\title{
La ética en la responsabilidad social empresarial
}

\section{Ethics in corporate social responsibility}

DOI: $10.46932 /$ sfjdv3n1-014

Received in: Dec 30st, 2021

Accepted in: Jan 1th, 2022

\author{
Karina Verónica Chávez Garcés \\ Associate Degree in Management and Marketing \\ ICPR-ABC Junior College \\ Economista de la Universidad de Guayaquil
}

Máster en Tecnología Educativa del Tecnológico de Monterrey

Vicerrectora del Instituto Superior Universitario Blue Hill College de Guayaquil, Ecuador.

E-mail:kchavez@bluehill.edu.ec

\author{
Maria Laura Roche Aguirre \\ Ingeniera Financiera de la Universidad Internacional SEK \\ Magister en Gobernabilidad y Gerencia Política de la Universidad Católica de Santiago de \\ Guayaquil en convenio con The School of Political Management of George \\ Washington University \\ PHD en Estudios Sociales en América Latina de la \\ Universidad Nacional de Córdoba, Argentina \\ Docente del Instituto Superior Universitario Blue Hill College de Guayaquil, Ecuador \\ E-mail: mroche@bluehill.edu.ec
}

\begin{abstract}
RESUMEN
La responsabilidad social empresarial se ha convertido en parte fundamental de la forma de hacer empresa, no solo conlleva satisfacer las necesidades financieras de la organización sino también ver cómo es sustentable para el entorno ya que debe contemplar los factores sociales, ambientales y económicos. Los cuales se resumen en 4 supuestos: el comportamiento empresarial, la ética en los negocios, las relaciones que se establezcan con los grupos de interés y las políticas empresariales a seguir. El presente estudio pretende identificar los factores que promueven la ética en los negocios sin dejar de lado la responsabilidad social. El estudio a aplicarse fue longitudinal y descriptivo. Se realizará a través de revisión bibliográfica y documental. Los resultados obtenidos demostraron que la técnica aplicada es una fuente extensa de las percepciones de las autoras, las que se utilizarán para futuras investigaciones.
\end{abstract}

Palabras clave: ética empresarial, ética en los negocios, responsabilidad social empresarial, negocios.

\begin{abstract}
Corporate social responsibility has become a fundamental part of the way of doing business, not only to satisfy the financial needs of the organization but also to see how it is sustainable for the environment since it must consider social, environmental and economic factors. These are summarized in 4 assumptions: business behavior, business ethics, the relationships established with stakeholders and the business policies to be followed. This study aims to identify the factors that promote business ethics without neglecting social responsibility. The study to be applied was longitudinal and descriptive. It will be carried out through bibliographic and documentary review. The results obtained showed that the applied technique is an extensive source of the authors' perceptions, which will be used for future research.
\end{abstract}


Keywords: business ethics, corporate ethics, corporate social responsibility, corporation, business.

\section{INTRODUCCIÓN}

¿Qué es hacer empresa hoy en día? A inicios del siglo 20, hacer empresa significaba ser parte de la revolución industrial, en palabras de Alvin Toffler, pertenecer a la tercera ola (1980). Actualmente, hace empresa conlleva más allá de producir un servicio o un producto. Es establecer un plan que abarca lo económico, político, social y ambiental. Por este motivo, ser responsable empresarialmente involucra a varios segmentos y grupos de interés, donde la empresa y sus funcionarios no son los únicos actores. La empresa debe ser responsable no solo con su cliente interno y externo, está llamada a serlo con el medio ambiente y la comunidad donde se desarrolla.

Para García, Rodríguez, Hernández y Martínez (2021) la RSE ha sido un proceso de concientización, que involucra o amalgama la ética, la moral, los recursos no solo materiales sino también humanos, lo que a su vez ha promovido que los consumidores hagan un análisis a la hora de ser parte del "ciclo empresarial" donde la empresa se convierte en un referente de "buenas acciones", lo cual es parte de hacer empresa, y una socialmente responsable.

EL posicionamiento de la RSE se debe a la acción en conjunto de varias instituciones y organismos internacionales creando una cadena de valor que promueve cada vez más las prácticas responsables en las empresas (Raufflet 2012). El modelo de Elkington (TBL) sigue vigente y con más fuerza a causa de las consecuencias ambientales que se han dado por intervención de las empresas.

Raufflet (2012) indica que el objetivo de las empresas actualmente es ser sustentables lo que implica no solamente “(...) mejorar las relaciones con los grupos de interés y reducir el impacto ambiental (...) sino también ser promotor del desarrollo humano” (p.33).

El objetivo de este estudio es describir cómo la RSE ha evolucionado a lo largo del tiempo y cómo se ha convertido en el eje principal del concepto de "hacer empresa". El documento consta de 4 partes, la primera hace un recorrido de su conceptualización, modelos y beneficios; la segunda presenta los resultados de su impacto; en la tercera parte se contrasta la ética y la RSE y la última parte presenta las conclusiones.

\section{ANTECEDENTES}

La Responsabilidad social empresarial (RSE) es uno de los retos actuales a los que se enfrenta la sociedad, que conduce al desarrollo sostenible del sistema socio-económico y a la propia sostenibilidad de las empresas. Cuando nos referimos a una empresa sostenible, hablamos de gestión de los factores sociales, ambientales y económicos, no solo que buscan resultados a corto plazo sino también a medio y 
largo plazo, lo hacen a la vez contribuyendo al bienestar de las generaciones presentes y futuras. La responsabilidad empresarial no es algo nuevo. John David Rockefeller creó su fundación, la Rockefeller Fundation, en 1913; y Andrew Carnegie en 1905. Pero el concepto en sí mismo nunca había sido concebido como parte integral de la organización, como una política corporativa, y un sistema organizado que permita desarrollar una serie de principios relacionados con la actividad de la empresa: Pensar en cómo la organización hace sus negocios.

Actualmente, la idea de responsabilidad corporativa está basada en cuatro principios:

1. Comportamiento empresarial, es lo que denominamos como el triple botton line,

2. Ética empresarial, cómo gestiono la empresa, cómo se hacen los productos, cómo se comercializan,

3. Relaciones empresariaes, identificación de todos los accionistas, públicos, audiencias, qué acciones se hacen para establecer relaciones con esos grupos, y

4. Política, establecimiento de una línea de acción en la compañía que establezca cómo se desarrollan los tres aspectos anteriores.

Lo que se denomina Triple botton line, o "triple base", implica la prosperidad económica, la integridad medioambiental y la responsabilidad social. Pero este concepto ha evolucionado desde los años'80. Se produjo una ampliación de la idea inicial de responsabilidad empresarial. La preocupación por la responsabilidad social empresarial ha pasado, en hora buena, a formar parte de la agenda de muchos directores en bastantes empresas. Es un importante reto, porque supone una forma más compleja de administrar y dirigir las empresas y organizaciones, de la que emanan nuevas exigencias: Transparencia, diálogo y múltiples acciones.

\section{CONCEPTUALIZACIÓN}

El Libro Verde de la Comisión Europea sobre Responsabilidad Social Empresarial (RSE) define que las empresas y organizaciones actuales se encuentran en unos entornos cada vez más complejos donde, en muchos casos, a su actividad económica tradicional deben añadir nuevas variables:

- Nuevas inquietudes y expectativas de los grupos de interés, clientes, poderes públicos, ciudadanos, inversores en una escala ya no local sino global,

- Decisiones de inversión o de compra de las personas e instituciones condicionadas cada vez más por criterios sociales,

- Preocupación sobre el deterioro medioambiental, y

- Necesidad de transparencia en las actividades empresariales

No cabe duda que las empresas contribuyen de una manera destacada al desarrollo social, pues, de hecho, su principal razón de ser es la generación de productos y servicios; lo que apareja la creación de 
puestos de trabajo, salarios e ingresos fiscales. Más allá de las actividades filantrópicas aisladas que algunas empresas han venido realizando en el tiempo, existen cada vez más ejemplos de planteamientos económicos, sociales y ambientales integrados y planificados como un elemento estratégico en la gestión de las organizaciones. Tanto por una creencia genuina en que las empresas podrían contribuir más o en otros aspectos a la sociedad, como por un planteamiento estratégico de imagen o reputación ante los grupos de interés, la responsabilidad social empresarial se ha situado "en la agenda" de las empresas y también de los decidores políticos y económicos.

La Norma UNE $165010^{1}$ "Ética. Sistema de gestión de la Responsabilidad Social Corporativa”, establece las siguientes definiciones:

Responsabilidad Social Corporativa: conjunto de obligaciones y compromisos legales y éticos, nacionales e internacionales con los grupos de interés, que se derivan de los impactos que la existencia, actividad y operación de las organizaciones producen en el ámbito social, laboral, medioambiental y de los derechos humanos. Dentro del concepto de RSC se incluye el de sostenibilidad que recoge los aspectos económicos, sociales, laborales, ambientales y de respeto de los derechos humanos.

Desarrollo sostenible: es el desarrollo que satisface las necesidades de la generación presente sin comprometer la capacidad de las generaciones futuras para satisfacer sus propias necesidades. Esta norma detalla los requisitos de la RSC en cuanto al comportamiento ante los accionistas, inversores, socios, donantes y representantes de la propiedad, ante los empleados, ante los clientes y usuarios, ante los suministradores y organizaciones participadas, ante la competencia, ante la administración y ante la Comunidad/Sociedad.

El Global Reporting Initiative (GRI), es un programa de Naciones Unidas que ha elaborado una "Guía para la elaboración de memorias de sostenibilidad sobre actuaciones económicas, medioambientales y sociales de la empresa". En este documento, que puede obtenerse en www.globalreporting.org, encontramos indicadores relacionados con las tres dimensiones del concepto de sostenibilidad y directrices para su correcta gestión:

\footnotetext{
${ }^{1}$ Desarrollada por la Asociación Española de Normalización y Certificación (AENOR) en 2009 en línea con el proceso de definición de la ISO 26000. Se trata de una guía con criterios para el mejor ejercicio de la Responsabilidad Social de la Empresa. https://observatoriorsc.org/norma-une-165010-ex/
} 
Tabla No.1 Indicadores de medición de sostebilidad empresarial"

\begin{tabular}{|c|c|l|}
\hline INDICADORES & \multicolumn{1}{|c|}{ CATEGORIA } & \multicolumn{1}{c|}{ ASPECTO } \\
\hline Económicos & Impactos económicos directos & $\begin{array}{l}\text { Clientes, Proveedores, Empleados, Proveedores } \\
\text { de Capital, Sector Público }\end{array}$ \\
\hline Ambientales & Ambientales & $\begin{array}{l}\text { Materias Primas, Energía, Agua, Biodiversidad, } \\
\text { Emisiones, vertederos, y residuos, proveedores, } \\
\text { productos y servicios, cumplimiento, transporte, } \\
\text { general }\end{array}$ \\
\hline Sociales & $\begin{array}{l}\text { Prácticas Laborales y trabajo } \\
\text { decente }\end{array}$ & $\begin{array}{l}\text { Empleo, Relaciones empresas/trabajadores, } \\
\text { Salud y seguridad, formación y educación, } \\
\text { diversidad y oportunidad }\end{array}$ \\
\hline \multicolumn{1}{|c|}{ Derechos Humanos } & $\begin{array}{l}\text { Estrategia y gestión, No discriminación, libertad } \\
\text { de asociación y negociación colectiva, trabajo } \\
\text { infantil, trabajo forzoso y obligatorio, medidas } \\
\text { de seguridad, derecho de los indígenas }\end{array}$ \\
\hline & Responsabilidad del producto & $\begin{array}{l}\text { Salud y seguridad del cliente, productos y } \\
\text { servicios, publicidad, respeto a la intimidad }\end{array}$ \\
\hline
\end{tabular}

Elaborado por: Autores

\section{LOS MODELOS DE EXCELENCIA}

Existen, a nivel mundial, numerosos Modelos de Excelencia desarrollados por entidades cuya misión es promover la mejora de las prácticas de gestión de empresas y otras organizaciones. Podemos citar, entre otros, al Modelo EFQM de Excelencia desarrollado por la European Foundation for Quality Management, el Iberoamericano desarrollado por FUNDIBEQ o el Malcolm Baldrige de USA. Han sido concebidos como una herramienta de diagnóstico (un marco de referencia para la gestión) que permite a las organizaciones identificar cuáles son sus puntos fuertes y áreas de mejora cuando se comparan con la "Excelencia". Todos estos Modelos contemplan la Responsabilidad Social de las organizaciones como uno de los Conceptos Fundamentales de la Excelencia que impregna los diferentes criterios o elementos que componen estos Modelos.

Figura No. 2 Diagrama de un modelo de organización que incluye RSE

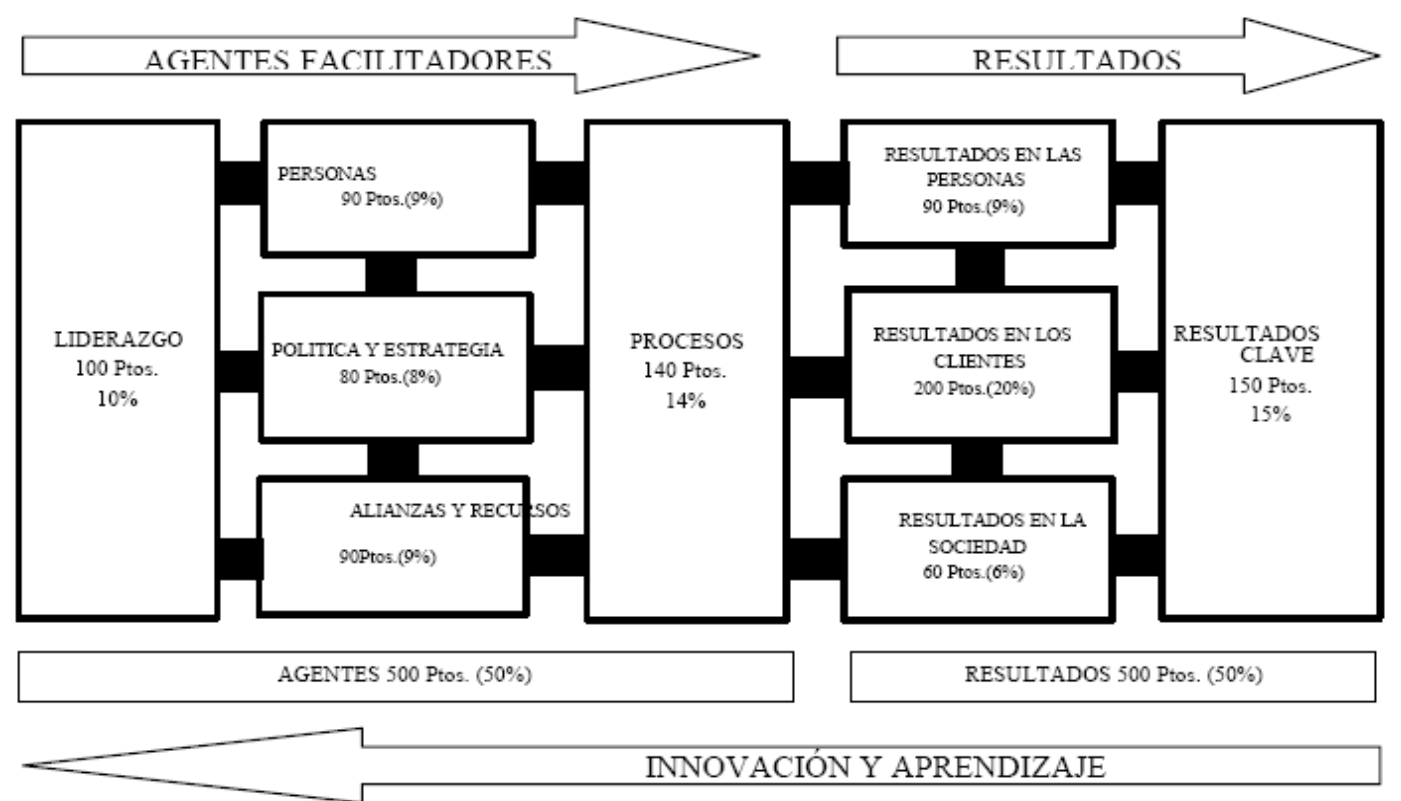

Elaborado por: Autores 
El Modelo de Excelencia de la EFQM está compuesto por nueve criterios diferenciándose claramente dos partes: el grupo formado por los llamados "Agentes Facilitadores" que se refieren a lo que la hace organización y cómo lo hace, y el grupo de criterios "Resultados" que dan cuenta de los logros obtenidos por la organización respecto a todos los grupos de interés (clientes, trabajadores, sociedad) y en relación a los objetivos globales (Maderuelo, 2002).

\subsection{LIDERAZGO}

- Desarrollar, actuando como modelo de referencia, los valores, los principios éticos y responsabilidades públicas que apoyan la cultura de la organización.

- Fomentar, apoyar y participar en actividades dirigidas a mejorar el medio ambiente a nivel global y la contribución de la organización a la sociedad, con vistas a respetar los derechos e intereses de las generaciones futuras.

- Ayudar y apoyar a las personas para hacer realidad sus planes, objetivos y metas.

- Fomentar y animar la igualdad de oportunidades y la diversidad.

- Comunicar los cambios y la razón de los mismos a las personas de la organización y otros grupos de interés.

\subsection{POLÍTICA Y ESTRATEGIA}

- Identificar, comprender y anticipar las necesidades y expectativas de los grupos de interés, actuales y futuros, incluidos clientes, empleados, partners, sociedad y accionistas.

- Analizar los datos relativos a las cuestiones sociales, medioambientales, de seguridad y legales, a corto y largo plazo.

- Equilibrar necesidades y expectativas a corto y largo plazo de todos los grupos de interés.

- Adecuar y desarrollar de manera continua estándares sociales y medioambientales con los partners.

\subsection{PERSONAS}

- Fomentar y garantizar la equidad en todo lo relacionado con el empleo, incluidas políticas, estrategias y planes de igualdad de oportunidades.

- Fomentar la concienciación e implicación en temas de higiene, seguridad, medio ambiente y responsabilidad social.

- Reconocer y tener en cuenta la diversidad y los distintos entornos culturales de procedencia fomentando actividades sociales y culturales. 
- Desarrollar, apoyar con tutores y formar a todas las personas para que se den cuenta y alcancen todo su potencial.

\subsection{ALIANZAS Y RECURSOS}

- Identificar las oportunidades para establecer alianzas clave con otras organizaciones y con la comunidad de acuerdo con la Política y Estrategia y la Misión de la organización. Diseñar la planificación económica y financiera y sus informes para desplegar en cascada las expectativas financieras de los grupos de interés a lo largo de la organización.

- Medir y gestionar cualquier impacto negativo de los activos de la organización en la comunidad y empleados (incluidas las cuestiones económicas, de higiene y seguridad).

- Utilizar los recursos de la organización de forma que no dañen el medio ambiente durante

- el ciclo completo de vida de un producto.

- Minimizar cualquier impacto global adverso de los productos, de la elaboración de los mismos y de los servicios.

- Desarrollar una tecnología innovadora y respetuosa con el medio ambiente (por ejemplo, que ahorre energía y recursos, reduzca al mínimo los residuos y emisiones y favorezca el reciclado y la reutilización).

\subsection{PROCESOS}

- Asesorar a los clientes sobre el uso responsable de los productos.

- Aplicar en la gestión de procesos estándares de sistemas como, por ejemplo, los de gestión de la calidad, gestión medioambiental o gestión de riesgos laborales.

- Anticipar e identificar mejoras en los productos y servicios de acuerdo con las futuras necesidades y expectativas de los clientes y otros grupos de interés.

\section{BENEFICIOS E IMPACTOS DE LA RSE PARA LAS EMPRESAS Y SU ENTORNO}

La integración de la responsabilidad social empresarial en la empresa puede producir una serie de efectos que de forma directa e indirecta la benefician.

\subsection{EFECTOS DIRECTOS}

- Mejora el entorno de trabajo

- Mayor compromiso de los trabajadores

- Incremento de la productividad y control de defectos 
- Reducción de costes (sostenibilidad económica)

- Mayor diálogo con las partes de interés y como resultado mejora de las relaciones con clientes, proveedores, comunidad local y administración.

- Mejora del medio ambiente.

\subsection{EFECTOS INDIRECTOS}

- Mejor percepción por parte del ciudadano y consumidores y mayor satisfacción del cliente.

- Mejora de la solidez de la empresa, ganando competitividad a nivel de mercado y controlando el riesgo reputacional.

- Mejora de las condiciones económicas de la comunidad, creación de negocio a largo plazo y en general favorecimiento del crecimiento del tejido empresarial.

- Mejores condiciones sociales de la comunidad, potenciación del consumo, la calidad de los trabajadores.

La medición del impacto se realiza desde distintos ángulos de la organización:

\subsection{RESULTADOS EN LOS CLIENTES}

- Realizar mediciones de la percepción que tienen los clientes acerca de la organización, así como otras medidas internas que permitan supervisar, entender, predecir y mejorar el rendimiento, en temas como el comportamiento proactivo, el perfil medioambiental, sellos de aprobación, marcas de garantía o etiquetas medioambientales

\subsection{RESULTADOS EN LAS PERSONAS}

- Realizar mediciones de la percepción que tienen los clientes acerca de la organización, así como otras medidas internas que permitan supervisar, entender, predecir y mejorar el rendimiento, en temas como la igualdad de oportunidades, el desarrollo de carreras profesionales, condiciones de higiene y seguridad, política e impacto medioambiental de la organización, papel de la organización en la comunidad y sociedad en general.

\subsection{RESULTADOS EN LA SOCIEDAD}

- Realizar mediciones de la percepción que tiene la sociedad acerca de la organización y se obtienen, por ejemplo, de encuestas, informes, artículos de prensa, reuniones públicas, representantes sociales y autoridades de la Administración, así como otros indicadores de rendimiento en temas tales como actuación de la organización como miembro responsable de la comunidad, incidencia 
en la economía local, nacional y mundial, reducir y evitar las molestias y daños provocados por sus actividades, preservación y mantenimiento de los recursos.

\subsection{RESULTADOS CLAVE}

- Realizar mediciones de los resultados clave definidos por la organización y acordados en su Política y Estrategia, en temas como rentabilidad, inversiones y activos, gestión y control presupuestario, cuota de mercado, número y valor añadido de las mejoras conjuntas realizadas con los partners.

\section{EJES ESTRATÉGICOS DE LA ÉTICA Y LA RESPONSABILIDAD SOCIAL EMPRESARIAL}

La RSE debe ser una iniciativa de marcado compromiso ético. Por tanto, en 1999 inicio un movimiento en pro de la RSE a nivel mundial, promovido por el Secretario de la ONU Koffi Annan, llegándose a firmar un Pacto Mundial. Lo que se promovía era la necesidad de alinear las políticas y prácticas corporativas con los valores y objetivos éticos universalmente aceptados. No es una certificación o un instrumento regulador, es una alianza internacional en torno a la responsabilidad social de las empresas con fines de fomentar una economía global más sustentable, incluyente, humana, y una nueva cultura empresarial.

El Pacto Mundial ubica a la RSE más allá de una preocupación filantrópica en tanto representa un compromiso voluntario basado en principios éticos de gestión empresarial, y el propósito de contribuir a la solución de retos sociales, que rebasan el horizonte inmediato de la empresa u organización. El Pacto comprende diez principios basados en acuerdos y convenios internacionalmente aceptados en cuatro áreas estratégicas ${ }^{2}$ :

1. Derechos humanos, a partir de la Declaración Universal de los Derechos Humanos en 1948,

2. Condiciones laborales, a partir de la Declaración de la Organización Internacional del trabajo sobre Principios y Derechos Fundamentales en el Trabajo de 1998,

3. Ambiente, a partir de la Declaración de Río de la Cumbre Internacional de las Naciones Unidas sobre Medio Ambiente y Desarrollo de 1992, y

4. Transparencia/anti-corrupción, a partir de la Convención de las Naciones Unidas contra la corrupción en 2004.

El Pacto Mundial es una realidad y aglutina a más de dos mil empresas y organizaciones en el mundo, incluyendo empresas pequeñas y medianas, además de empresas se encuentran organizaciones

\footnotetext{
${ }^{2}$ Pacto Mundial https://www.pactomundial.org
} 
sindicales, empresariales, universidades, organizaciones no gubernamentales, asociaciones no lucrativas, entre otras (Limón, 2006)

Para citar ejemplos palpables se mencionan los casos de España, para el año 2002 se habían adherido ciento sesenta empresas y organizaciones; en Chile en octubre del 2004 firmaron su adhesión más de seiscientos mil empresarios mis pymes de los sectores primario, secundario y terciario. En México, en el 2005 tuvo lugar el acto de adhesión del país al Pacto Mundial. Otros ejemplos que muestran la preocupación por la RSE en el mundo está en agrupaciones como Fórum Empresa, en el continente americano; Corporate Social Responsibility Campaign de la Unión Europea; Social Enterprise Knowledge Network de Estados Unidos, Latinoamérica y Europa liderado por la Harvard Business School; también se ubican ejemplos en países como Alemania, Austria, Gran Bretaña, España, Suiza.

\section{RSE Y ÉTICA EN LA PRÁCTICA}

Que una empresa haga acción social no quiere decir que sea una compañía responsable. La responsabilidad social se refiere a los compromisos, estrategias y prácticas operativas que una compañía desarrolla para gestionar su comportamiento corporativo, ética corporativa y sus relaciones con los diferentes accionistas.

○ ¿Qué se entiende por responsabilidad en la empresa, a qué denominamos responsabilidad corporativa?

○ ¿Cómo puede ser gestionada la responsabilidad corporativa?

○ ¿Debería ser comunicada; cómo?

Se puede identificar históricamente tres formas generales de gestionar la responsabilidad:

- Como conducta altruista/ filantrópica, suele perseguir el fin de mejorar la imagen del propietario o de la compañía. No responde a planteamientos generales de la empresa. Por ejemplo, el fenómeno de las fundaciones expresa, muchas veces, esta conducta.

○ Como estrategia de marketing, busca lograr diferenciación. Ejemplo: Marketing Verde

- Como filosofía de la organización, como parte de la política de la organización. Se trata de ser un ciudadano corporativo.

Como podemos observar, estamos ante dos planteamientos opuestos respecto de la RSE y que Robbins y Coulter, denominan la posición clásica orientada a la maximización de las ganancias y la posición socioeconómica; dirigida a la protección y mejoramiento del bienestar de la sociedad. A esto se agrega la polarización que de la interpretación de la RSE se ha hecho, generando entonces dos corrientes: Desde la perspectiva del altruismo, o desde la perspectiva de la obligación y responsabilidad primordial basada en principios y compromisos. 
En la ciudad de Guayaquil se realizó una breve encuesta a un grupo de propietarios de empresas, profesionales y empleados se diversos sectores para revisar cuánto conocían sobre los aspectos de la RSE, entre los principales hallazgos se tiene:

- El 77\% de los encuestado afirmo conocer sobre la definición de la RSE, el $40 \%$ de los encuestados provienen de empresas de manufacturas, mientras que los demás se reparten equitativamente entre actividades de comercio, servicios profesionales, educativos, transporte, alimentos. El $90 \%$ de las empresas encuestadas facturan anualmente menos de $\$ 100,000$ y el $10 \%$ más de $\$ 1,000,000$.

- El 67\% de las empresas consideran que la RSE es muy importante para la organización mientras que el $22 \%$ lo considera no tan importante y el $11 \%$ no lo considera importante. A su vez el $67 \%$ de las empresas no cuentan con prácticas de RSE y el 33\% las tienen en forma parcial.

- Entre los principales factores identificados para implementar estas prácticas señalan el desconocimiento de las metodologías y se consideran una pequeña empresa para hacerlo.

- Lo que esperan el 60\% de los encuestados implementar en un futuro es la seguridad y salud laboral, los derechos humanos y la gestión del cliente.

Son evidentes son las manifestaciones de este movimiento en favor de la responsabilidad social, pero desde luego que no ha de ser una tarea fácil si consideramos la otra cara del problema, es decir, en tanto se considere a la ética empresarial como obstáculo para hacer negocio: Se centre la misión en beneficios medidos en dinero, poder y prestigio y se le considere simplemente como un efecto cosmético. Hablar de la RSE implica referirse a la ética, a la que debemos considerar como su fundamento sustantivo en tanto le provee de herramientas para tratar la complejidad moral en la toma de decisiones.

En ese sentido cabe destacar lo dicho por Hill y Jones (2010) con referencia a la RSE: "cuando las empresas evalúan decisiones desde una perspectiva ética, debe haber una presunción que favorezca la adopción de cursos de acción que aumenten el bienestar de la sociedad en general"; más aún, debemos entender que la manera en que una empresa alcanza sus objetivos, "cae dentro de la esfera de la ética" como dicen Sisk y Sverdlik.

Sin embargo, las implicaciones de la ética van más allá, implica identificar cuáles son los bienes propios de la actividad empresarial que se han de proporcionar a la sociedad y que le dan sentido, la legitiman, -llamados bienes internos- para estar en condiciones de definir las metas a perseguir, los valores y los hábitos necesarios a incorporar para alcanzar tales metas, a este conjunto de implicaciones es lo que se denomina ética aplicada.

Tiene relación como la empresa reconoce, trata, maneja el impacto económico que tiene sobre su entorno. La única razón por la cual una empresa existe es hacer buenos productos, comercializarlos correctamente y generar beneficios crecientes. Esta concepción de responsabilidad significa que el deber, 
la máxima preocupación de la empresa es contentar al accionista No se cuestiona el hecho de que una empresa tenga que hacer bien su trabajo, tenga que comercializar buenos productos. El tema es cómo se hacen los negocios, la ética de los negocios es un principio básico. La responsabilidad comienza cuando se cumple con las actividades de la empresa más allá de las exigencias legales existentes al respecto. La empresa no sólo está orientada a su relación con los accionistas sino con todos los grupos que tienen alguna especie de vinculación con la compañía, hay preocupación por el entorno en el que se vive.

Si bien promulgar la RSE puede contribuir a generar una buena reputación corporativa, no garantiza una actuación éticamente correcta en el proceder de la empresa. Por ejemplo, se puede citar el caso paradigmático en que se ilustra esta afirmación, Enron, empresa, admirada por su éxito en lo relativo al tema, pero cuya buena prensa durante años por ser "buena ciudadana" no fue óbice para conocer el desastroso final, de infeliz recuerdo, que conmovió a la opinión pública mundial, dejó sembrado el camino de cinismo, de pérdidas millonarias, de ruina económica y trufado de miseria moral; además de la pérdida de miles de puestos de trabajo y alguna que otra sentencia de cárcel de añadidura. Todo ello, claro es, respuesta de una más que evidente falta de ética en la gestión.

En cuanto a la ética y con la ecológica es uno de los problemas mayores que enfrenta la región latinoamericana es el deterioro acelerado de los recursos naturales, acompañado de la contaminación urbana y rural. La creciente deforestación, la erosión de suelos, la sequía y la pérdida de la biodiversidad son indicadores de un manejo inadecuado de nuestra herencia natural. Igualmente, los consumidores en mercados globales exigen crecientemente productos y procesos menos contaminantes y más amistosos al ambiente. La responsabilidad ambiental es la imputabilidad de una valoración positiva o negativa por el impacto ecológico de una decisión. Se refiere generalmente al daño causado a otras especies, a la naturaleza en su conjunto o a las futuras generaciones, por las acciones o las no-acciones de otro individuo o grupo. Ejemplo: "La responsabilidad ambiental de las empresas petroleras es grande debido a la contaminación del mar y las playas provocada por los derrames".

Hans Jonas (2010) propone un imperativo que, siguiendo formalmente el imperativo categórico kantiano, ordena: "obra de tal modo que los efectos de tu acción sean compatibles con la permanencia de una vida humana auténtica en la Tierra". Dicho imperativo se conoce como el "principio de responsabilidad" y es de gran importancia en ecología y derecho ambiental. La responsabilidad ambiental recae tanto en los individuos, como en las empresas, países y en la especie humana en su conjunto.

En cuanto a la ética política algunos sectores de izquierda son críticos de las corrientes que promueven la RSE por considerar que ésta sirve sobre todo como una forma de publicidad empresarial. Ellos destacan la paradoja de empresas nocivas para la salud o para el ambiente que pretenden mejorar su reputación mediante un discurso retórico acerca de la RSC, por ejemplo, una empresa de cigarrillos que compra el tabaco a campesinos pobres para contrarrestar las críticas sobre el cáncer de pulmón. Algunos 
economistas de derecha, como Milton Friedman (Bour, 2012), consideran que "la única responsabilidad social de la empresa debe ser la generación de ganancias en favor de sus dueños, accionistas y trabajadores en un marco de competencia libre y abierta. Si la empresa sacrificase ganancias en nombre de la responsabilidad social -piensa Friedman- sería menos atractiva para inversionistas, trabajadores y consumidores, y a la larga perecería frente a los competidores.

La RSE no constituye necesariamente una forma de responsabilidad jurídica, porque bien puede no estar prevista en las leyes y descansar simplemente en la libre decisión de las empresas. Sin embargo, incluso cuando no está respaldada en la ley, la violación de un compromiso social puede costarles a las compañías fuertes críticas en la opinión pública a través de los medios de prensa, e incluso una pérdida de accionistas. Algunos estudios de caso ilustran la manera cómo una empresa suele tener interés comercial en ser socialmente responsable. Ello constituye una respuesta a la crítica de Friedman, pues es posible obtener más ganancias al administrar de manera socialmente responsable. En las empresas modernas, la Responsabilidad Social Corporativa, es más que un conjunto de prácticas puntuales, es más bien un programa integrado y reconocido de prácticas y políticas socialmente responsables, que soporta la toma de decisiones y conlleva al éxito del negocio a largo plazo.

La empresa está vinculada con muchos grupos de los cuales depende, por lo tanto, tiene que atender a esas relaciones y ser responsable socialmente significa ser responsable en esas relaciones, con esos grupos, para que la empresa pueda insertarse en la sociedad, ser un ciudadano corporativo. Los canales de comunicación son dialógicos, es primordial generar diálogo, relación con los grupos con los cuales la empresa tiene que trabajar. La comunicación es concebida como herramienta estratégica para construir la responsabilidad, contrariamente a la comunicación como herramienta táctica.

Existen dos investigaciones realizadas en Estados Unidos en la segunda mitad del siglo pasado, una respecto de la ética en los negocios realizada por el Reverendo Raymond Baumhart, realizando un Doctorado en la Universidad de Harvard, y otra en el terreno de los valores personales desarrollada por George W. England, publicada en el Journal of the Academy of Management en marzo de 1967; ambos concluyen que los valores personales de los ejecutivos determinan las normas éticas de una empresa, sus objetivos y estrategias corporativos, así como del conjunto de empresas agrupadas en un sector. Estas conclusiones encuentran similitud con Sisk \& Sverdlik; y Sánchez, (2015) cuando afirman que "los sistemas de valores personales determinan para un individuo, lo que está bien o incorrecto, lo que es bueno o malo, exitoso o fallido, placentero o desagradable, o cualquier otra evaluación bipolar similar”.

Entonces ahora la atención se dirige hacia el referente que el tomador de decisiones considere, si se queda en el ámbito estrictamente personal. Si no apela a la conciencia moral alcanzada por la sociedad, al núcleo de valores morales mínimos compartidos por la sociedad en que se inserta, conocido como ética cívica, o si no lo circunscribe a la actividad empresarial, porque cada tipo de organización, ya bien sea 
una universidad, un partido político, un hospital, un negocio, se legitima de manera diferenciada ante la sociedad en tanto son distintas las necesidades sustantivas a satisfacer.

El problema con el referente es que, en el mejor de los casos, en palabras de Koontz y Weihrich (1998), “los administradores responderán a los valores aprobados por la sociedad y darán prioridad a los tenidos en mayor estima". En última instancia, en la empresa debe definirse un marco moral explicitado y clarificado que sirva de referente para la toma de decisiones, estará influenciado por los valores personales, pero deberá estar determinado por la conciencia moral de la sociedad.

\section{CONCLUSIONES}

Dado que la ética "es esencialmente un saber para actuar de un modo racional ... y tiene por tarea mostrarnos cómo deliberar bien con objeto de hacer buenas elecciones", la ética proporciona dos modos de orientar racionalmente la toma de decisiones con prudencia, que implica la capacidad de hacer elecciones en consonancia con los fines últimos, elegir los medios más adecuados conlleva a tomar decisiones prudentes, y justicia, que toma de referente al derecho vigente, y las convicciones morales imperantes para calificar los medios elegidos, lo cual a su vez conlleva a decisiones justas

Sin embargo, no es suficiente tomar tales referentes únicamente, se debe averiguar que valores y derechos han de ser racionalmente respetados, toda vez que por el hecho de que una norma haya sido promulgada por los procedimientos legales establecidos, no implica necesariamente que sea justa.

La concepción de la responsabilidad social empieza a ampliarse, empiezan a surgir conceptos como respuesta social corporativa, comportamiento social corporativo y, lo más actual que se maneja es el concepto de ciudadanía corporativa. La empresa, más allá del triple botton line, lo que tiene que hacer es introducir mecanismos de respuesta, de ajuste, con la sociedad, es decir, políticas corporativas que definan cómo se trabaja la responsabilidad social. Está bien tener una conducta responsable, pero hay que empezar a definir políticas corporativas que encaucen esa actividad.

La Responsabilidad Social Corporativa y la Ética Empresarial no son sinónimos intercambiables. Son todos los más campos semánticos muy próximos, tangentes, pero no sustitutivos. Pero ya es un punto de partida hablar de RSE; una forma de entrar al sistema para estimularlo hacia metas más elevadas, a partir de la cual avanzar en la búsqueda de la excelencia, y el buen hacer económico y moral por parte de la empresa. Podría interpretarse, a la RSE como un pre requisito, una condición de posibilidad, algo necesario, pero no suficiente; como algo sin lo cual resultaría muy difícil actuar éticamente o, lo que es más sutil, encajar la ética en los fundamentos de la empresa.

Dentro de este contexto, es importante resaltar que conlleva una tarea de empresas como de la sociedad, el tener responsabilidad ética; construir una sociedad más próspera y un mundo más habitable. Y esto, en sí mismo, ya es bueno y éticamente loable. 
Por consiguiente, si se quiere explotar a fondo las posibilidades que en ellas se puede apreciar el camino hacia una nueva sociedad, no puede ser otra la decisión de cimentarlas en una cultura corporativa caracterizada por valores firmes, criterios claros, principios sólidos, en definitiva, por la esencia de la Ética Empresarial. 


\section{REFERENCES}

C. K. Prahalad y Michael E. Porter, Harvard Business Review on Corporate Responsibility. Comisión Europea. (2001). Libro Verde: Fomentar un marco europeo para la responsabilidad social de las empresas. https://observatoriorsc.org/libro-verde-fomentar-un-marco-europeo-para-laresponsabilidad-social-de-las-empresas/

Elkington, J. (1997). Cannibals with forks: The triple bottom line of 21 first century business. Oxford. García Amado,J.,Rodríguez Izaguirre,M., Hernández Reséndez,Z., Martínez Rocha,V.(2021). Influencia de la responsabilidad social en la decisión de compra de los consumidores de ciudad Victoria. South Florida Journal of Development, Miami, v.2, n.2, p.2770-2780. Recuperado de https://southfloridapublishing.com/ojs/index.php/jdev/article/view/395/369

Global Reporting Initiative (GRI) impulsado por Naciones Unidas. Fundamentos (2016) https://www.globalreporting.org/standards/media/1439/spanish-gri-101-foundation-2016.pdf

Hill, Charles y Gareth, Jones (2010). Administración estratégica un enfoque integrado, Editorial McGraw-Hill Interamericana S.A., Sexta Edición, Santafé de Bogotá-Colombia, pp. 54

Kate Soper, What is Nature? Culture, Politics and the Non-Human (Blackwell, Oxford, 1995)

Koontz y Weihrich. (1998) Administración, una perspectiva global. 11a. Edición. Edit. Mc Graw Hill, México. Obtenido de "http://es.wikipedia.org/wiki/Responsabilidad_ambiental"

Kotler, Philip y Lee, Nancy, Corporate Social Responsibility. Doing the most good for your company and your cause, John Wiley \& Sons, New Jersey, 2005.

Limón Suárez, Enrique. (2004) Análisis de la no contratación de personal en pymes del sector servicios de Veracruz Periodo 2002-2003. Tesis para obtener el grado de Maestro en Administración, Universidad Cristóbal Colón, Veracruz. Recuperado en 27 de octubre de 2021, de https://www.eumed.net/rev/tecsistecatl/n0/els1.htm

Maderuelo Fernández, J. A. (2002). Gestión de la calidad total: El modelo EFQM de excelencia. Medifam, 12(10), 41-54. Recuperado en 27 de octubre de 2021, de http://scielo.isciii.es/scielo.php?script=sci_arttext\&pid=S1131-57682002001000004\&lng=es\&tlng=es.

Modelo EFQM de Excelencia https://geoinnova.org/blog-territorio/modelo-europeo-excelenciaempresarial-

fqm/?gclid=Cj0KCQjwlOmLBhCHARIsAGiJg7ktZQbNWS88Xw50VVNssF36b_SaI4pHwctE4A3MSo1jFZB1_c4CHAaAvGkEALw_wcB

Norma UNE 165010 Ética Sistema de gestión de la RSC (AEC, 2009).

https://observatoriorsc.org/norma-une-165010-ex/

Perdiguero, Tomás G., La responsabilidad social de las empresas en un mundo global, Anagrama, Barcelona, 2003.

Raufflett, E. (2012). Responsabilidad Social Empresarial. 1ra.edición. Pearson. México

Robbins, S.\& Coulter, M. (20212). Administración. 11va. edición. Pearson. México. 
Sánchez Jiménez, Vicente. (2015). Influencia entre conductas sociales y decisiones empresariales. Artículo Política y Sociedad. Universidad Complutense de Madrid. Recuperado el 20 de octubre de 2021, de

https://go.gale.com/ps/i.do?id=GALE\%7CA422327676\&sid=googleScholar\&v=2.1\&it=r\&linkaccess=a bs\&issn=11308001\&p=IFME\&sw=w\&userGroupName=anon\%7Eaf19d8b6

Sisk y Sverdlik. ( 1979) Administración y Gerencia de empresas. Edit. South-Western Publishing Co. USA.

Stoner, Freeman y Gilbert Jr. (1996) Administración. 6ª Edición. Edit. Prentice Hall, México. Tofler, A.(1980). The third wave. Morrow\&Co. New York. 